\title{
Influence of anti-osteoporosis treatments on the incidence of COVID- 19 in patients with non-inflammatory rheumatic conditions
}

\author{
Josep Blanch-Rubió ${ }^{1,2,}{ }^{*}$, Natalia Soldevila-Domenech 3,7,*, Laura Tío², Jone Llorente-Onaindia², \\ Manuel Ciria-Recasens ${ }^{1,2}$, Luciano Polino ${ }^{2}$, Alba Gurt ${ }^{5}$, Rafael de la Torre ${ }^{3,6,7}$, Rafael \\ Maldonado ${ }^{2,4, \S}$, Jordi Monfort ${ }^{1,2, \S}$, and the Covidmar Study Group ${ }^{\#}$ \\ ${ }^{1}$ Rheumatology Service, Hospital del Mar, Passeig Marítim, Barcelona 08003, Spain \\ ${ }^{2}$ IMIM (Hospital del Mar Medical Research Institute), PRBB, Barcelona 08003, Spain \\ ${ }^{3}$ Integrative Pharmacology and Systems Neuroscience Research Group, Neurosciences Research Program, IMIM- \\ Institut Hospital del Mar d'Investigacions Mèdiques, PRBB, Barcelona 08003, Spain \\ ${ }^{4}$ Laboratory of Neuropharmacology, Department of Experimental and Health Sciences, Universitat Pompeu Fabra, \\ PRBB, Barcelona 08003, Spain \\ ${ }^{5}$ CAP Vila Olímpica, Parc Sanitari Pere Virgili, Barcelona 08003, Spain \\ ${ }^{6}$ Spanish Biomedical Research Centre in Physiopathology of Obesity and Nutrition (CIBERObn), Instituto de Salud \\ Carlos III (ISCIII), Madrid 28029, Spain \\ ${ }^{7}$ Department of Experimental and Health Sciences, Universitat Pompeu Fabra, PRBB, Barcelona 08003, Spain \\ *Equal contribution \\ ${ }^{\S}$ Equally supervised this work \\ \#A list of authors and their affiliations appears in Acknowledgments and in Collaborators section of PubMed. \\ Correspondence to: Rafael Maldonado; email: rafael.maldonado@upf.edu \\ Keywords: denosumab, zoledronate, calcium, vitamin D, anti-resorptive drugs, COVID-19 \\ Received: July 21, $2020 \quad$ Accepted: September 9, $2020 \quad$ Published: October 20, 2020
}

Copyright: ( 2020 Blanch-Rubió et al. This is an open access article distributed under the terms of the Creative Commons Attribution License (CC BY 3.0), which permits unrestricted use, distribution, and reproduction in any medium, provided the original author and source are credited.

\section{ABSTRACT}

Coronavirus disease 19 (COVID-19) is currently a global pandemic that affects patients with other pathologies. Here, we investigated the influence of treatments for osteoporosis and other non-inflammatory rheumatic conditions, such as osteoarthritis and fibromyalgia, on COVID-19 incidence. To this end, we conducted a crosssectional study of 2,102 patients being treated at the Rheumatology Service of Hospital del Mar (Barcelona, Spain). In our cohort, COVID-19 cumulative incidence from March 1 to May 3, 2020 was compared to population estimates for the same city. We used Poisson regression models to determine the adjusted relative risk ratios for COVID-19 associated with different treatments and comorbidities. Denosumab, zoledronate and calcium were negatively associated with COVID-19 incidence. Some analgesics, particularly pregabalin and most of the studied antidepressants, were positively associated with COVID-19 incidence, whereas duloxetine presented a negative association. Oral bisphosphonates, vitamin D, thiazide diuretics, anti-hypertensive drugs and chronic nonsteroidal anti-inflammatory drugs had no effect on COVID-19 incidence in the studied population. Our results provide novel evidence to support the maintenance of the main anti-osteoporosis treatments in COVID-19 patients, which may be of particular relevance to elderly patients affected by the SARS-CoV-2 pandemic.

\section{INTRODUCTION}

Infections by SARS-CoV-2, a novel coronavirus that emerged in China in late 2019 [1], and the disease that it causes, COVID-19, became a global pandemic on March $11^{\text {th }}, 2020$ [2]. By July19 ${ }^{\text {th }}, 2020$, COVID-19 had infected 14,348,475 people and caused 603,167 deaths worldwide [3]. The incidence of COVID-19 is 
heterogeneous within different countries. In Spain, the area of Barcelona (Catalonia, Spain) has suffered one of the highest rates of incidence and deaths in Europe, mostly between March and April of 2020 [4].

COVID-19 initially has a viral phase with symptoms that include fever, dry cough, anosmia/ageusia, odynophagia and diarrhea, among others. Approximately seven days after this initial phase, some patients develop a systemic pro-inflammatory state and progress to more severe symptoms, such as dyspnea, shortness of breath, pulmonary infiltrates and hypoxemia. The progression of the disease has been associated with a hyper inflammatory response with high levels of inflammatory markers and proinflammatory cytokines, sometimes accompanying a pro-coagulation state. Patients following this evolution sometimes become seriously ill, often requiring admission to Intensive Care Units, and some ultimately may die [5].

The innate and acquired immune responses play a crucial role in the progression of the disease. The immune system seems to be dysregulated in severe forms of COVID-19, probably due to abnormal responses by monocytes, macrophage, and/or dendritic cells [6]. Some disease-modifying, anti-rheumatic drugs used in the treatment of immune-mediated inflammatory diseases may have a protective effect, such as the IL6 antagonists tocilizumab and sarilumab [7], which are currently off-label used to treat patients. Our team (article under revision) and other researchers [8-10] have also shown that some of these antirheumatic treatments reduce COVID-19 incidence.

Among the diseases treated by rheumatology, osteoporosis is an age-related chronic disease that affects tens of millions people worldwide, requiring long-term treatment [11]. It is a global chronic pandemic causing enormous morbidity, mortality and economic burdens [12]. The possible effects of antiosteoporosis pharmacologic treatments in the clinical expression and incidence of COVID- 19 remain unknown. Nonetheless, the most prominent organizations for rheumatology and bone studies, such as the American College of Rheumatology (ACR) [13], the European League Against Rheumatism (EULAR) [14], the American Society for Bone and Mineral Research (ASBMR) [15], and the International Osteoporosis Foundation (IOF) [16], currently do not recommend discontinuing the administration of rheumatologic drugs to treat COVID-19 due to their likely neutral effects. However, such recommendations are based on expert opinions and, to our knowledge, no data are available regarding the safety of using such drugs to treat COVID-19.
Determining whether anti-osteoporosis treatments are safe for COVID-19 patients and whether they influence COVID-19 incidence and its clinical expression could positively impact patient prognosis. These questions apply to all anti-osteoporosis compounds including denosumab, a fully human monoclonal antibody against RANK-L, that inhibits osteoclastogenesis. Denosumab is widely used to treat osteoporosis, but also to prevent skeletal-related events in advanced malignancies with bone metastasis [17]. The RANKL/RANK system participates in processes related to the immune system, including lymph-node development, lymphocyte differentiation, dendritic cell survival and T- cell activation, and tolerance induction [18]. Furthermore, osteoprotegerin, a natural decoy with similar effects to those of denosumab on RANK-L, may elicit beneficial effects to patients suffering from viral infections [19]. Therefore, denosumab may modulate the immune response associated to viral infections, such as SARS$\mathrm{CoV}-2$.

Non-inflammatory rheumatic conditions such as osteoporosis, osteoarthritis and fibromyalgia are all characterized by a high incidence of chronic pain and are mainly treated with classical non-steroidal antiinflammatory drugs (NSAIDs) and opioids, but also with gabapentinoids and two particular antidepressant drugs, duloxetine and amitriptyline. In addition to chronic pain, these pathological conditions often present co-morbid emotional disorders that are treated with antidepressants. However, there is no data on the potential effects of these drugs on COVID-19 incidence.

Here, in order to elucidate the possible effects of antiosteoporosis drugs (anti-resorptives, calcium and vitamin D) and associated treatments (analgesics and antidepressants) on COVID-19 incidence and clinical expression, we carried out a cross-sectional study of the cumulative incidence of COVID-19 in rheumatic patients suffering from non-inflammatory conditions and living in the influence area of a referral hospital in Barcelona, Spain.

\section{RESULTS}

A total of 2,498 individuals were examined for eligibility and 2,102 fulfilled the inclusion criteria and were included in the analysis, $80.5 \%$ of which were women. Table 1 shows the description of the studied population and the distribution of COVID-19 across studied variables. The mean age was 66.4 years (SD, 13.3 ) and $63.7 \%$ of the population had osteoarthritis, $43.5 \%$ osteoporosis and $27.2 \%$ fibromyalgia. The most prevalent coexisting conditions were hypertension (42.4\%), pulmonary disease $(15.0 \%)$, cardiovascular (CV) disease (14.9\%) and diabetes (12.6\%). Regarding 
Table 1. Characteristics of the study population and distribution of confirmed or hsCOVID-19 cases.

\begin{tabular}{|c|c|c|c|}
\hline \multirow{2}{*}{ Characteristic } & \multirow{2}{*}{$\begin{array}{c}\text { All population } \\
(\mathrm{N}=2102)\end{array}$} & \multicolumn{2}{|c|}{ Confirmed or hsCOVID-19 } \\
\hline & & No $(N=1993)$ & Yes $(\mathrm{N}=109)$ \\
\hline Men & $409(19.5 \%)$ & $388(19.5 \%)$ & $21(19.3 \%)$ \\
\hline Women & $1693(80.5 \%)$ & $1605(80.5 \%)$ & $88(80.7 \%)$ \\
\hline Age $[$ mean $(\mathrm{SD})]$ & $66.4(13.3)$ & $66.5(13.3)$ & $65.7(13.2)$ \\
\hline \multicolumn{4}{|c|}{ Non-inflammatory rheumatic diagnosis ${ }^{1}$} \\
\hline Osteoarthritis & $1340(63.7 \%)$ & $1263(63.4 \%)$ & $77(70.6 \%)$ \\
\hline Osteoporosis & $914(43.5 \%)$ & $880(44.2 \%)$ & $34(31.2 \%)$ \\
\hline Fibromyalgia & $571(27.2 \%)$ & $539(27.0 \%)$ & $32(29.4 \%)$ \\
\hline \multicolumn{4}{|l|}{ Coexisting conditions } \\
\hline Diabetes & $264(12.6 \%)$ & $245(12.3 \%)$ & $19(17.4 \%)$ \\
\hline Hypertension & $892(42.4 \%)$ & $845(42.4 \%)$ & $47(43.1 \%)$ \\
\hline Pulmonary disease & $315(15.0 \%)$ & $290(14.6 \%)$ & $25(22.9 \%)$ \\
\hline CV disease & $314(14.9 \%)$ & $286(14.4 \%)$ & $28(25.7 \%)$ \\
\hline Cancer or active treatment & $121(5.76 \%)$ & $115(5.77 \%)$ & $6(5.50 \%)$ \\
\hline Chronic kidney disease & $114(5.42 \%)$ & $104(5.22 \%)$ & $10(9.17 \%)$ \\
\hline History of organ transplantation & $9(0.43 \%)$ & $6(0.30 \%)$ & $3(2.75 \%)$ \\
\hline Any of these comorbidities & $1232(58.6 \%)$ & $1159(58.2 \%)$ & $73(67.0 \%)$ \\
\hline \multicolumn{4}{|l|}{ Treatments followed } \\
\hline Denosumab & $264(12.6 \%)$ & $256(12.8 \%)$ & $8(7.34 \%)$ \\
\hline Intravenous Zoledronate & $179(8.52 \%)$ & $173(8.68 \%)$ & $6(5.50 \%)$ \\
\hline Oral bisphosphonates & $143(6.80 \%)$ & $136(6.82 \%)$ & $7(6.42 \%)$ \\
\hline Teriparatide & $25(1.19 \%)$ & $25(1.25 \%)$ & $0(0.00 \%)$ \\
\hline Calcium & $490(23.3 \%)$ & $474(23.8 \%)$ & $16(14.7 \%)$ \\
\hline Vitamin D & $1303(62.0 \%)$ & $1241(62.3 \%)$ & $62(56.9 \%)$ \\
\hline Thiazide diuretics & $262(12.5 \%)$ & $248(12.4 \%)$ & $14(12.8 \%)$ \\
\hline SERMs & $11(0.52 \%)$ & $11(0.55 \%)$ & $0(0.00 \%)$ \\
\hline Analgesics & $1220(58.0 \%)$ & $1154(57.9 \%)$ & $66(60.6 \%)$ \\
\hline Gabapentin & $164(7.80 \%)$ & $153(7.68 \%)$ & $11(10.1 \%)$ \\
\hline Pregabalin & $146(6.95 \%)$ & $134(6.72 \%)$ & $12(11.0 \%)$ \\
\hline Opioids & $546(26.0 \%)$ & $510(25.6 \%)$ & $36(33.0 \%)$ \\
\hline Other Analgesics & $959(45.6 \%)$ & $906(45.5 \%)$ & $53(48.6 \%)$ \\
\hline Antidepressants & $657(31.3 \%)$ & $612(30.7 \%)$ & $45(41.3 \%)$ \\
\hline Tricyclic antidepressants & $124(5.90 \%)$ & $116(5.82 \%)$ & $8(7.34 \%)$ \\
\hline Amitriptyline & $102(4.85 \%)$ & $94(4.72 \%)$ & $8(7.34 \%)$ \\
\hline Others & $22(1.05 \%)$ & $22(1.10 \%)$ & $0(0.00 \%)$ \\
\hline Dual-action antidepressants & $277(13.2 \%)$ & $260(13.0 \%)$ & $17(15.6 \%)$ \\
\hline Duloxetine & $207(9.85 \%)$ & $198(9.93 \%)$ & $9(8.26 \%)$ \\
\hline Venlafaxine & $60(2.85 \%)$ & $53(2.66 \%)$ & $7(6.42 \%)$ \\
\hline Others & $10(0.48 \%)$ & $9(0.45 \%)$ & $1(0.92 \%)$ \\
\hline SSRIs antidepressants & $333(15.8 \%)$ & $307(15.4 \%)$ & $26(23.9 \%)$ \\
\hline Reboxetine & $2(0.10 \%)$ & $2(0.10 \%)$ & $0(0.00 \%)$ \\
\hline Trazodone & $33(1.57 \%)$ & $31(1.56 \%)$ & $2(1.83 \%)$ \\
\hline Glucocorticoids & $60(2.85 \%)$ & $53(2.66 \%)$ & $7(6.42 \%)$ \\
\hline Inhaled Glucocorticoids & $189(8.99 \%)$ & $172(8.63 \%)$ & $17(15.6 \%)$ \\
\hline Anti-hypertensive drugs & $646(30.7 \%)$ & $610(30.6 \%)$ & $36(33.0 \%)$ \\
\hline ACE inhibitors & $363(17.3 \%)$ & $344(17.3 \%)$ & $19(17.4 \%)$ \\
\hline ARBs & $290(13.8 \%)$ & $273(13.7 \%)$ & $17(15.6 \%)$ \\
\hline Chronic NSAIDs & $318(15.1 \%)$ & $301(15.1 \%)$ & $17(15.6 \%)$ \\
\hline Synthetic DMARDs & $30(1.43 \%)$ & $26(1.30 \%)$ & $4(3.67 \%)$ \\
\hline Biologic DMARDs & $1(0.05 \%)$ & $1(0.05 \%)$ & $0(0.00 \%)$ \\
\hline
\end{tabular}




\section{COVID-19 status}

Grade of hsCOVID-19 symptomatology

Mild

Moderate

$\begin{array}{ccc}63(3.00 \%) & \text { NA } & 63(57.8 \%) \\ 16(0.76 \%) & \text { NA } & 16(14.7 \%) \\ 30(1.43 \%) & \text { NA } & 30(27.5 \%) \\ 71(3.38 \%) & \text { NA } & 71(65.1 \%) \\ 25(1.19 \%) & \text { NA } & 25(22.9 \%) \\ 3(0.14 \%) & \text { NA } & 3(2.75 \%) \\ 1(0.05 \%) & \text { NA } & 1(0.92 \%) \\ 9(0.43 \%) & \text { NA } & 9(8.26 \%) \\ 38(1.81 \%) & \text { NA } & 38(34.9 \%) \\ 13(0.62 \%) & \text { NA } & \\ 23(1.09 \%) & \text { NA } & 13(11.9 \%) \\ 48(2.28 \%) & \text { NA } & 23(21.1 \%) \\ & \text { NA } & 48(44.0 \%)\end{array}$

Severe

COVID-19 Evolution:

Home

Hospitalization

$25(1.19 \%)$

$25(22.9 \%)$

NIV

ICU

$1(0.05 \%)$

Death

Positive SARS-CoV-2 test (PCR)

$9(0.43 \%)$

Radiography:

Pathologic unilateral

$23(1.09 \%)$

$48(44.0 \%)$

COVID-19 diagnosed by PCR or radiography

${ }^{1}$ Some individuals have more than one diagnosis.

$\mathrm{ACE}=$ angiotensin-converting enzyme. $\mathrm{ARBs}=$ angiotensin II receptor blockers. $\mathrm{CV}=$ cardiovascular disease. DMARDs = disease modifying anti-rheumatic drugs. hsCOVID-19 = highly suspected COVID-19 cases. ICU = intensive care unit. NA = not applicable. NIV = non-invasive ventilation. NSAIDs = non-steroid anti-inflammatory drugs. $P C R=$ polymerase chain reaction. SERMs = selective estrogen receptor modulator. $\mathrm{SD}=$ standard deviation. SSRIs = selective serotonin reuptake inhibitors.

treatments, $62 \%$ were treated with vitamin $\mathrm{D}, 23.3 \%$ with calcium, $12.6 \%$ with denosumab, and $8.5 \%$ with intravenous zoledronate. More than a half of the population was exposed to analgesics and almost a third to antidepressants, mainly serotonin reuptake inhibitors (SSRIs) (15.8\%) and dual-action antidepressants $(13.2 \%)$.

A total of 109 individuals had COVID-19 diagnosis (hereafter, COVID-19-positive or "COVID-19+" patients), representing $5.19 \%$ of the individuals included. They presented a higher prevalence of diabetes, CV disease, pulmonary disease and chronic kidney disease than those not diagnosed with COVID-19 (hereafter, COVID-19-negative "COVID-19-" patients). In terms of treatments, the exposure to denosumab, intravenous zoledronate, vitamin $\mathrm{D}$, and selective estrogen receptor modulators (SERMs) was lower for COVID-19+ than for COVID-19- patients, whereas antidepressant treatment was more frequent in COVID-19-positive individuals.

As shown in Table 2, the age-standardized cumulative incidence rate in our population was $4.68 \%$ (CI95\% $3.78-5.59 \%$ ), being slightly higher than that in the general population of Barcelona (3.69\%; CI95\% 3.66-3.73\%). However, when stratifying by the presence of osteoporosis, osteoarthritis and fibromyalgia, patients with osteoporosis presented lower rates $(2.98 \%$, CI95\% $1.88-4.08)$ than the general population, whereas patients with osteoarthritis (4.58\%, CI95\% 3.46-5.70) and fibromyalgia $(4.45 \%, \quad$ CI95\% $2.76-6.14)$ showed slightly higher rates.
Adjusted associations between different exposure variables (clinical characteristics and treatments) and COVID-19 diagnosis are shown in Table 3. CV disease was the comorbidity showing the highest RR of COVID19 (RR=1.84; CI95\%1.17-2.87), followed by chronic kidney disease, diabetes and pulmonary disease. Patients suffering from cancer or in active cancer treatment did not show an increased RR for COVID-19 diagnosis. Regarding treatments, the RR for COVID-19 was 0.58 (CI95\%0.28-1.22) for denosumab, 0.62 (CI95\%0.271.41) for intravenous zoledronate and 0.64 (CI95\%0.371.12) for calcium. No association between COVID-19 and oral bisphosphonates, vitamin $\mathrm{D}$ or thiazide diuretics was found. Analgesics, particularly pregabalin $(R R=1.55$; CI95\%0.86-2.79), gabapentin ( $\mathrm{RR}=1.39$; CI95\%0.752.58) and opioids ( $R R=1.25$; CI95\%0.85-1.83) showed an increased RR for COVID-19. In the case of antidepressants, SSRIs presented an RR of 1.54 (CI95\%1.00-2.36). The tricyclic antidepressant amitriptyline presented an RR of 1.38 (CI95\% 0.7, 2.71) and the RR of all dual-action antidepressants together was 1.22 (CI95\% 0.72, 2.08). In sharp contrast, the RR of the dual-action antidepressant duloxetine was 0.68 (CI95\% 0.34-1.34). Figure 1 summarizes the adjusted RR for the incidence of COVID-19 according to the exposure to the most prevalent studied treatments.

Finally, the RR estimates using propensity score matching for the exposure to denosumab, intravenous zoledronate and calcium are included in Supplementary Table 3. The resulting RR estimates were almost equivalent to the unmatched database, with values of 
Table 2. Crude and age-adjusted cumulative incidence rates of confirmed or hsCOVID-19 cases in our cohort and in the population of Barcelona (reference population) registered from March 1st to May 3rd, 2020, stratified by the diagnosis of osteoporosis, osteoarthritis and fibromyalgia.

\begin{tabular}{|c|c|c|c|c|c|c|c|c|c|}
\hline \multirow[b]{3}{*}{$\begin{array}{l}\text { Age } \\
\text { group } \\
\text { (years) }\end{array}$} & \multirow[b]{3}{*}{$\begin{array}{c}\text { Incidence rate of } \\
\text { confirmed or } \\
\text { hsCOVID-19 cases in } \\
\text { Barcelona }\end{array}$} & \multicolumn{8}{|c|}{ Confirmed or hsCOVID-19 cases in our cohort } \\
\hline & & \multicolumn{2}{|c|}{ All population } & \multicolumn{2}{|c|}{ Population with osteoporosis } & \multicolumn{2}{|c|}{ Population with osteoarthritis } & \multicolumn{2}{|c|}{ Population with fibromyalgia } \\
\hline & & $\begin{array}{c}\text { Crude } \\
\text { cumulative } \\
\text { incidence rate }\end{array}$ & $\begin{array}{c}\text { Age- } \\
\text { adjusted } \\
\text { cumulative } \\
\text { incidence } \\
\text { rate } \\
\end{array}$ & $\begin{array}{c}\text { Crude } \\
\text { cumulative } \\
\text { incidence rate }\end{array}$ & $\begin{array}{c}\text { Age- } \\
\text { adjusted } \\
\text { cumulative } \\
\text { incidence } \\
\text { rate }\end{array}$ & $\begin{array}{c}\text { Crude } \\
\text { cumulative } \\
\text { incidence rate }\end{array}$ & $\begin{array}{l}\text { Age-adjusted } \\
\text { cumulative } \\
\text { incidence } \\
\text { rate }\end{array}$ & $\begin{array}{c}\text { Crude } \\
\text { cumulative } \\
\text { incidence rate }\end{array}$ & $\begin{array}{c}\text { Age-adjusted } \\
\text { cumulative } \\
\text { incidence rate }\end{array}$ \\
\hline $20-29$ & $4818 / 195194(2.47 \%)$ & $0 / 0(0 \%)$ & $0 \%$ & $0 / 0(0 \%)$ & $0 \%$ & $0 / 0(0 \%)$ & $0 \%$ & $0 / 0(0 \%)$ & $0 \%$ \\
\hline $30-39$ & $6628 / 250517(2.65 \%)$ & $2 / 44(4.55 \%)$ & $0.84 \%$ & $0 / 5(0 \%)$ & $0 \%$ & $0 / 10(0 \%)$ & $0 \%$ & $2 / 31(6.45 \%)$ & $1.19 \%$ \\
\hline $40-49$ & $7515 / 255707(2.94 \%)$ & $11 / 172(6.40 \%)$ & $1.20 \%$ & $1 / 20(5.00 \%)$ & $0.94 \%$ & $5 / 61(8.20 \%)$ & $1.54 \%$ & $6 / 116(5.17 \%)$ & $0.97 \%$ \\
\hline $50-59$ & $7807 / 218163(3.58 \%)$ & $28 / 409(6.85 \%)$ & $1.10 \%$ & $6 / 110(5.45 \%)$ & $0.87 \%$ & $18 / 220(8.18 \%)$ & $1.31 \%$ & $15 / 208(7.21 \%)$ & $1.15 \%$ \\
\hline $60-69$ & $5061 / 177078(2.86 \%)$ & $26 / 545(4.77 \%)$ & $0.62 \%$ & $8 / 234(3.42 \%)$ & $0.44 \%$ & $20 / 371(5.39 \%)$ & $0.70 \%$ & $6 / 147(4.08 \%)$ & $0.53 \%$ \\
\hline $70-79$ & $5147 / 143113(3.60 \%)$ & $23 / 545(4.22 \%)$ & $0.44 \%$ & $8 / 295(2.71 \%)$ & $0.28 \%$ & $19 / 391(4.86 \%)$ & $0.51 \%$ & $3 / 52(5.77 \%)$ & $0.61 \%$ \\
\hline $80-89$ & $8581 / 97289(8.82 \%)$ & $15 / 327(4.59 \%)$ & $0.33 \%$ & $7 / 208(3.37 \%)$ & $0.24 \%$ & $12 / 252(4.76 \%)$ & $0.34 \%$ & $0 / 10(0 \%)$ & $0 \%$ \\
\hline $90+$ & 4795 / $25924(18.5 \%)$ & $4 / 47(8.51 \%)$ & $0.16 \%$ & $4 / 38(10.53 \%)$ & $0.20 \%$ & $3 / 32(9.38 \%)$ & $0.18 \%$ & $0 / 1(0 \%)$ & $0 \%$ \\
\hline All & $3.69 \%$ & $5.19 \%$ & $4.68 \%$ & $3.72 \%$ & $2.98 \%$ & $5.75 \%$ & $4.58 \%$ & $5.60 \%$ & $4.45 \%$ \\
\hline (CI95\%) & $(3.66-3.73 \%)$ & $(4.24-6.13 \%)$ & $(3.78-5.59 \%)$ & $(2.49-4.95 \%)$ & $(1.88-4.08 \%)$ & $(4.5-6.99 \%)$ & $(3.46-5.70 \%)$ & $(1.71-9.50 \%)$ & $(2.76-6.14 \%)$ \\
\hline
\end{tabular}

hsCOVID-19 = highly suspected COVID-19.

Cl95\% $=95 \%$ confidence intervals.

Table 3. Adjusted Relative Risk (aRR) with 95\% confidence intervals (CI95\%) of confirmed or hsCOVID-19 according to the presence of several comorbidities and treatments.

\begin{tabular}{|c|c|c|c|}
\hline & N (All = 2102) & Model 1 - aRR (CI95\%) & Model 2 - aRR (CI95\%) \\
\hline Women & 1693 & $1.12(0.71,1.76)$ & $1.11(0.7,1.76)$ \\
\hline Age & & $0.99(0.98,1.01)$ & $0.99(0.98,1.01)$ \\
\hline \multicolumn{4}{|l|}{ Comorbidities } \\
\hline CV disease & 314 & $1.84(1.17,2.87)$ & $1.86(1.19,2.91)$ \\
\hline Diabetes & 264 & $1.2(0.71,2.03)$ & $1.19(0.7,2.03)$ \\
\hline Pulmonary disease & 315 & $1.36(0.76,2.45)$ & $1.34(0.73,2.46)$ \\
\hline Chronic kidney disease & 114 & $1.58(0.82,3.07)$ & $1.56(0.8,3.03)$ \\
\hline Cancer or active treatment & 121 & $1.06(0.46,2.46)$ & $1.06(0.45,2.47)$ \\
\hline \multicolumn{4}{|l|}{ Treatments followed } \\
\hline Denosumab & 264 & $0.58(0.28,1.22)$ & $0.59(0.28,1.23)$ \\
\hline Intravenous Zoledronate & 179 & $0.62(0.27,1.41)$ & $0.61(0.27,1.38)$ \\
\hline Oral bisphosphonates & 143 & $0.97(0.45,2.08)$ & $0.97(0.46,2.06)$ \\
\hline Calcium & 490 & $0.64(0.37,1.12)$ & $0.64(0.37,1.11)$ \\
\hline Vitamin D & 1303 & $0.92(0.63,1.36)$ & $0.91(0.62,1.34)$ \\
\hline Thiazide diuretics & 262 & $0.95(0.54,1.67)$ & $0.94(0.53,1.66)$ \\
\hline Analgesics & 1220 & $0.92(0.61,1.38)$ & \\
\hline Gabapentin & 164 & & $1.39(0.75,2.58)$ \\
\hline Pregabalin & 146 & & $1.55(0.86,2.79)$ \\
\hline Opioids & 546 & & $1.25(0.85,1.83)$ \\
\hline Other Analgesics & 959 & & $0.94(0.64,1.37)$ \\
\hline Dual-action antidepressants & 277 & $1.22(0.72,2.08)$ & \\
\hline Duloxetine & 207 & & $0.68(0.34,1.34)$ \\
\hline Tricyclic antidepressants & 124 & $1.06(0.54,2.08)$ & \\
\hline Amitriptyline & 102 & & $1.38(0.7,2.71)$ \\
\hline
\end{tabular}




\begin{tabular}{llll} 
SSRIs antidepressants & 333 & $1.54(1,2.36)$ & $1.39(0.9,2.14)$ \\
Inhaled Glucocorticoids & 189 & $1.42(0.73,2.77)$ & $1.39(0.7,2.74)$ \\
Anti-hypertensive drugs & 646 & $1.06(0.7,1.6)$ & \\
ACE inhibitors & 363 & & $0.98(0.58,1.65)$ \\
ARBs & 290 & $0.94(0.57,1.56)$ & $1.05(0.62,1.76)$ \\
Chronic NSAIDs & 318 & $0.95(0.58,1.55)$ \\
\hline
\end{tabular}

$\mathrm{ACE}=$ angiotensin-converting enzyme. $\mathrm{ARBs}=$ angiotensin II receptor blockers. CV = cardiovascular disease. hsCOVID-19= highly suspected COVID-19 cases. NSAIDs = non-steroid anti-inflammatory drugs. SSRIs = selective serotonin reuptake inhibitors.

0.73 (CI95\% 0.30- 1.78) for denosumab, 0.55 (CI95\% $0.20-1.44)$ for intravenous zoledronate and 0.72 (CI95\% 0.39-1.37) for calcium. The adjusted RRs by the other treatments did not differ from the crude ones: 0.87 (CI95\% 0.30-2.52) for denosumab, 0.43 (CI95\% $0.37-1.35$ ) for intravenous zoledronate and 0.67 (CI 95\% 0.36-1.27) for calcium.

\section{DISCUSSION}

The present study reveals that the main treatments currently used for osteoporosis are not associated with an increase in COVID-19 incidence. All the treatments analyzed in our study were continued after the presentation of COVID-19 symptoms following the

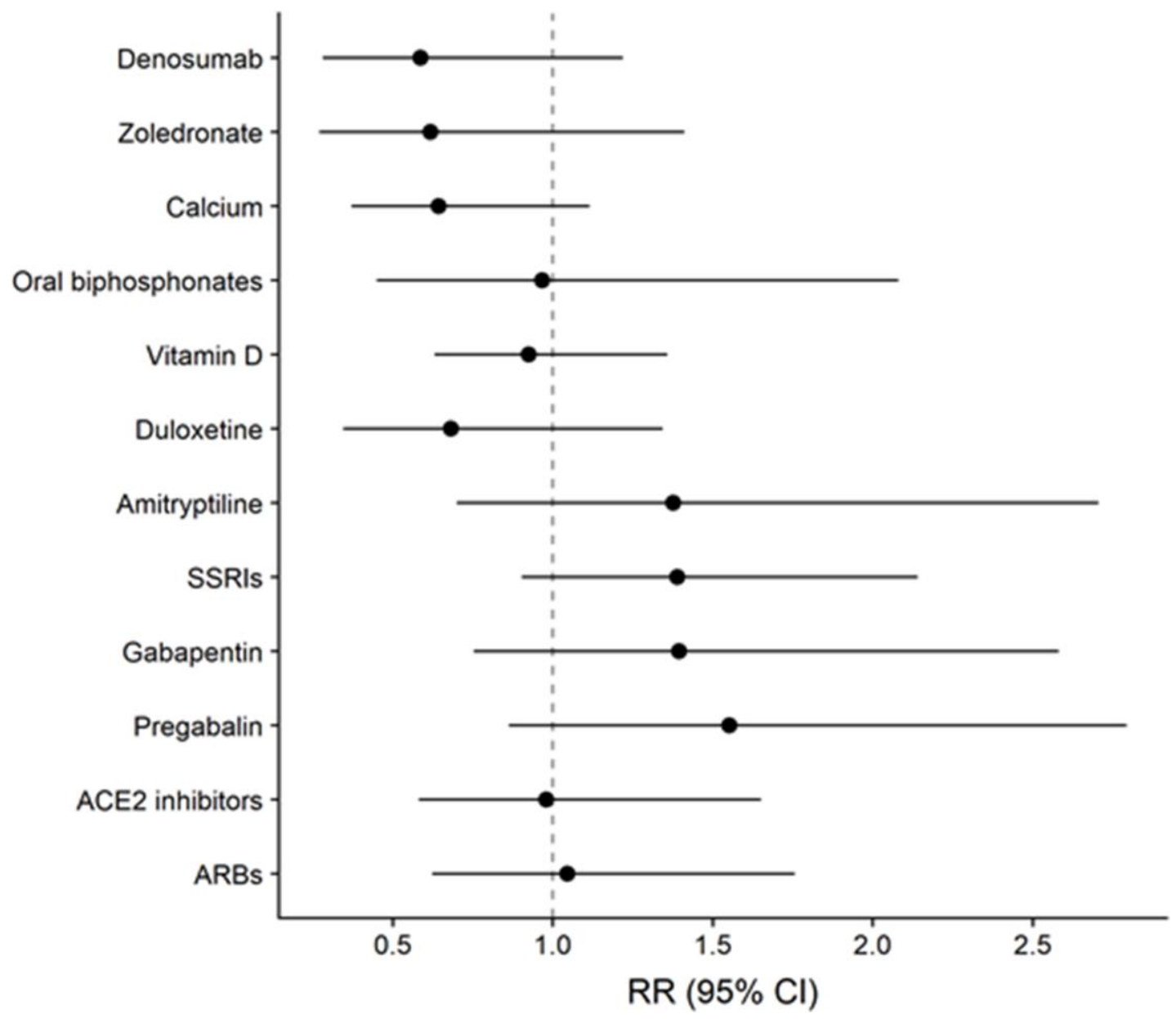

Figure 1. Relative Risk (RR) with 95\% Confidence Interval (CI95\%) of COVID-19 diagnosis according to the exposure to different treatments, adjusted by sex, age, CV disease, diabetes, pulmonary disease, chronic kidney disease and active cancer or treatments. The effect of Denosumab Zoledronate, Calcium, Oral bisphosphonates and Vitamin D were obtained from Model 1. Estimates for Duloxetine, SSRIs, Gabapentin, Pregabalin, ACE inhibitors and ARBs were obtained from Model 2. 
recommendations of multiple international rheumatology $[13,14]$ and bone field organizations $[15,16]$. Interestingly, the exposure to two anti-resorptive drugs, denosumab (RR=0.58; $\mathrm{CI} 95 \% \quad 0.28,1.22)$ and intravenous zoledronate $(\mathrm{RR}=0.62$; CI95\% $0.27,1.41)$, was associated with a 40\% decreased risk of COVID-19. Recent relevant studies underline the high predictive value of the RR points estimate in these Poisson regression models, which often present high confidence interval ranges [20, 21].

The anti-osteoporosis target of denosumab is the RANK/RANKL system involved in the inhibition of osteoclastogenesis. The RANK/RANK-L system also participates in immune responses, including lymph-node development, lymphocyte differentiation, dendritic cell survival and T-cell activation [18]. Denosumab prevents RANK-L from binding to RANK receptor, thereby inhibiting osteoclast differentiation. RANK-L inhibition by denosumab modifies immune cell profiles and decreases the activity of pro-inflammatory cytokines [22]. This decrease in the inflammatory responses might elicit beneficial effects during viral infections, as previously reported with other RANK-L inhibitors [19], and could explain the decreased incidence of COVID-19 cases among patients treated with denosumab. Indeed, COVID-19 progression has been associated with a hyperactivity of pro-inflammatory cytokines [5] and a dysregulation of the immune system related to an abnormal monocytes/macrophage/dendritic cells response [6], which could be attenuated by denosumab treatment.

In contrast to denosumab, bisphosphonates bind to hydroxyapatite crystals and inhibit mature osteoclast function through induction of apoptotic pathways or blockade of cytoskeletal assembly by inhibition of lipid modification of associated proteins [23]. The exposure to intravenous zoledronate, but not to other oral bisphosphonates, showed a negative association with the incidence of COVID-19. The higher potency of intravenous zoledronate in comparison to any other oral bisphosphonate used in this study [24] may explain the differential result obtained with both groups of bisphosphonates. Intravenous zoledronate treatment reduces mortality after hip fracture [25], which may be related to decreased risk of $\mathrm{CV}$ disease, general health status improvement, fracture prevention, improved regulation of the immune system and a reduced incidence of pneumonia [26, 27]. Interestingly, zoledronate may make dendritic cells and their precursors less susceptible to SARS-CoV-2 infection, which could explain the beneficial effects here reported on COVID-19 incidence [28]. Indeed, zoledronate inhibits the prenylation of small GTPases [29, 30], which may hinder endosomal exocytosis in the dendritic cells required for the advance of SARS-CoV-2 infection. These protective effects on dendritic cells and their precursors may lead to immune-stimulation of $\mathrm{T}$ cell expansion and enhanced activity of natural killer cells, crucial mechanisms to prevent the progression of SARS-CoV-2 infection in the lung [31].

In the present study, patients treated with calcium supplements also presented a decreased risk of COVID19 ( $R R=0.64$, CI95\% 0.37, 1.12). In agreement, recent studies have reported reduced COVID-19 mortality in patients treated with calcium [32]. A decrease in total and ionized calcium blood levels has been reported in COVID-19 patients [33]. Changes in calcium levels in COVID-19 patients may be due to alterations in intestinal absorption, imbalance in regulatory mechanism involving parathyroid hormone and vitamin $\mathrm{D}$, or to a direct effect caused by SARS- CoV-2 [33]. However, our results are compatible with no relevant effect of vitamin D on COVID-19 incidence ( $R R=0.97$, CI95\% 0.45, 2.08). A possible mechanism that may explain the beneficial effects of calcium in COVID-19 found in our study could be related to the action of calcium, through a specific calcium-based signal, on the generation of two immune cell types: $T$ follicular helper cells and $\mathrm{T}$ follicular regulatory cells. These $\mathrm{T}$ cells promote an appropriate immune response against infectious agents, such as viruses [33, 34]. Accordingly, calcium supplements may counteract the decreased serum levels of calcium promoted by SARS-CoV-2 infection, which may lead to an improvement of the immune cell response and attenuate the probability of infection progression.

In agreement with the protective effects shown by the exposure to the main anti-osteoporosis treatments, ageadjusted cumulative incidence of COVID-19 in osteoporosis patients was lower ( $\mathrm{RR}=2.98 \%$, CI95\% $1.88,4.08)$ than in all the patients included in this study ( $\mathrm{RR}=4.68 \%$, CI95\% 3.78-5.59\%). This decrease was not observed with other non-inflammatory rheumatic conditions analyzed in our study. The comorbidity that produced the highest positive association with COVID19 incidence in non-inflammatory rheumatic conditions was $\mathrm{CV}$ disease ( $\mathrm{RR}=1.84, \mathrm{CI} 95 \%$ 1.17, 2.87;), although other co-morbidities, such as chronic kidney disease ( $R R=1.58, C I 95 \% 0.82,3.07)$ also substantially enhanced this incidence, as expected.

In our study, the exposure to different antidepressant drugs produced various effects on COVID-19 incidence. Interestingly, the dual acting serotonin/norepinephrine inhibitor duloxetine decreased the incidence of COVID19 ( $\mathrm{RR}=0.68, \mathrm{CI} 95 \%$ 0.34, 1.34). Antidepressant drugs have been postulated to modulate immune responses by modifying the serotonin/norepinephrine equilibrium, 
which modifies the balance of the different $\mathrm{T}$ cell populations involved in the release of cytokines [35]. Antidepressants that change this serotonin/ norepinephrine balance, such as the dual inhibitor duloxetine, may facilitate the maintenance or restauration of an appropriate $\mathrm{T}$ cells equilibrium and cytokine production [35]. However, amitriptyline, a tricyclic antidepressant that also has this dual serotonin/norepinephrine inhibitory effect, showed a different profile than duloxetine on the incidence of COVID-19. Both antidepressants have a completely different activity on other receptors [36], including antagonist activity of duloxetine on sigma-1 receptors [37], a mechanism postulated as a target of interest for re-purposing compounds for COVID-19 treatment [38]. Interestingly, SSRIs cause bone loss by a mechanism that counteracts local anti-resorption [39], in contrast to anti-osteoporosis medications that decrease COVID-19 incidence in our study. Also, opposite to antiosteoporosis medications, patients taking SSRIs presented a 50\% enhanced risk of COVID-19 $(\mathrm{RR}=1.54$, CI95\% 1.0, 2.36).

Our results suggest a positive association between the exposure to gabapentinoids and COVID-19 incidence. This increased risk was mainly revealed after pregabalin exposure ( $\mathrm{RR}=1.55, \mathrm{CI} 95 \%$ 0.86, 2.79), an antiepileptic drug mainly used in our population for chronic pain treatment. Pregabalin predominantly blocks the alpha2-delta subunit of voltage-gated calcium channels [40] and decreases immune responses under chronic pain conditions [41]. However, chronic pregabalin administration in HIV patients increased $\mathrm{T}$ cell levels in blood suggesting a possible activation of the immune response under this particular condition [42]. Moreover, SARS-CoV-2 binds to angiotensin-converting-enzyme2 (ACE2) receptors and pregabalin has been reported to decrease these receptors in animal models [43]. When the amount of ACE2 is reduced due to the virus occupancy, individuals could be more susceptible to severe COVID-19 illness because enough ACE2 is still available for viral entry, whereas this decreased ACE2 availability facilitates angiotensin II-mediated injury. This pathophysiological mechanism associated to reduced ACE2 expression may promote inflammation, cell death and organ failure, mainly in the heart and lungs [44]. This mechanism may also contribute to the increased risk of COVID-19 we observed here among patients treated with pregabalin.

Our results from the two groups of anti-hypertensive drugs analyzed, ACE2 inhibitors and angiotensin II receptor blockers (ARBs), are compatible with no effect on the incidence of COVID-19. These results are in agreement with a recent meta-analysis showing that the use of these anti-hypertensive drugs in patients with
COVID-19 does not increase the risk of SARS-CoV-2 infection and COVID-19 severity, being these treatments associated with a decreased risk of mortality [45]. Therefore, all these results suggest that treatment with ACE2 and ARBs should be continued in COVID19 patients who are taking these anti-hypertensive medications.

Some limitations of this study must be addressed. Osteoporosis grade and related comorbidities may have biased the risk estimates in our study. To control for the potential effect of confounding by indication, RRs of COVID-19 were also estimated after propensity score matching with the main available covariates that predict receiving denosumab, zoledronate or calcium treatment and the RRs obtained were also negatively associated with COVID-19. The similar results obtained with denosumab, zoledronate and calcium using both analyses suggest that the effects of these antiosteoporosis medications on COVID-19 were not due to the presence of osteoporosis or underlying comorbidities. Furthermore, the data in this crosssectional study were collected from a large number of patients. Thus, data have been collected by different researchers and some data may have been missed or slight differences in classification criteria may have been applied. Notwithstanding, all of the researchers were expert clinicians or medical researchers, and several meetings were held among them to unify classification criteria and to review clinical records. Also due to the cross-sectional design of the study, the degree of severity of some patients may have changed throughout the survey and these changes may not have been assessed. Additionally, our study cohort included patients from a tertiary hospital that were probably suffering from more severe forms of non-inflammatory rheumatic conditions in comparison with patients in primary or secondary care settings, which may introduce bias. However, all the anti-osteoporosis medications were uniformly covered by the public health insurance system in Spain, thus avoiding biases related medication costs. Finally, some asymptomatic patients may not have been registered due to the low availability of tests for SARS-Cov-2 in our country in the early stages of the pandemic.

In summary, our results reveal that chronic treatment with some of the main anti-osteoporosis drugs currently available, anti-resorptives, calcium and vitamin $\mathrm{D}$, are not associated with increased risk of COVID-19. In contrast, a decreased incidence of COVID-19 was revealed with two anti-resorptives drugs, denosumab and zoledronate, as well as with calcium treatment. Some of the pain treatments used in these noninflammatory rheumatic conditions may influence COVID-19 outcomes, since the incidence of COVID-19 
was decreased in patients treated with duloxetine and increased in those taking pregabalin. In conclusion, our data are consistent with a lack of direct relationship between osteoporosis therapies and COVID-19 incidence, providing scientific evidence in support of the recently-published guidelines by the ACR, EULAR, ASBMR and IOF [13-16] to maintain anti-osteoporosis treatments for COVID-19 patients, which were based solely on expert opinions.

\section{MATERIALS AND METHODS}

\section{Study design, and population}

A cross-sectional study was performed at the Rheumatology Service of Hospital del Mar (Barcelona, Spain) that includes patients diagnosed with osteoporosis, osteoarthritis and/or fibromyalgia. Patients receiving care at the outpatient Rheumatology Service for the last six months were eligible. The exclusion criteria were $<18$ years old, previous death not related with SARS- CoV-2 infection, presence of immunemediated inflammatory disease, a negative SARS-CoV2 test, or failing to follow up at the primary care center during the studied period.

\section{Outcomes}

Hospital and primary care clinical history revision have been performed and the patient data included in this study were collected from March $1^{\text {st }}$ to May $3^{\text {rd }}, 2020$, the period of the highest COVID-19 incidence in Spain. The primary outcome was the presence of COVID-19 diagnosis, although other related variables were also recorded, including PCR results, lung radiography, symptomatology and evolution. At the time of revision, demographic and clinical data were also collected, with a particular focus on comorbidities (Supplementary Table 1) and medical drug prescriptions (Supplementary Table 2).

\section{Statistical analysis}

Cumulative incidence was adjusted for age by direct standardization using a COVID-19 epidemiological database (RSAcovid19) generated by the Department of Health of the Government of Catalonia [39]. This database contains daily cumulative positive cases and daily cumulative suspicious cases activated by the epidemiological surveillance service. For this analysis, we selected positive or suspicious cases registered from March $1^{\text {st }}$ to May $3^{\text {rd }}, 2020$ in the city of Barcelona, which was the reference population for direct standardization [40]. The RSAcovid19 database considers as positive the cases that tested positive in a diagnostic test (PCR, rapid test, or ELISA test), and those confirmed by an epidemiologist as a positive case while they consider as "suspicious cases" those who had symptoms classified by a health professional as a possible case, but without a diagnostic test.

To evaluate the associations between different treatments (with >100 exposed patients; reference category: unexposed) and the presence of COVID-19, Poisson regression models with robust variance estimation were used to estimate RR and 95\% confidence intervals (CI95\%). Models were adjusted by sex, age, diabetes, pulmonary disease, cardiovascular disease, chronic kidney disease, and active cancer or treatment. Model 1 included the following treatments: denosumab, oral/intravenous bisphosphonates, calcium, vitamin D, thiazide diuretics, analgesics, antidepressants (dual action vs tricyclic vs SSRIs), inhaled glucocorticoids, anti- hypertensive drugs and NSAIDs. Model 2 included the specific effect of the analgesics gabapentin, pregabalin, opioids and others; the dual-action antidepressant duloxetine; the tricyclic antidepressant amitriptyline; and two types of anti-hypertensive drugs: ACE2 inhibitors and ARBs; together with denosumab, oral/intravenous bisphosphonates, calcium, vitamin D, thiazide diuretics, inhaled glucocorticoids and chronic NSAIDs.

Finally, the presence of osteoporosis, together with the studied comorbidities (sex, age, cardiovascular disease, diabetes, pulmonary disease, kidney disease and cancer) were used to calculate the probability of treatment assignment for denosumab, bisphosphonates and calcium with propensity score matching based on the nearest neighbor method [41]. Therefore, each treated individual was matched with an untreated individual whose propensity score was closest to that of the treated subject. Statistical analyses were performed using R (R Foundation for Statistical Computing, Vienna, Austria) version 3.5.2.

\section{Ethics statement}

All research in this study was conducted in accordance with the ethical standards of the Declaration of Helsinki and according to national and international guidelines. The observational study was approved by the Parc de Salut Mar Ethical Committee on Clinical Studies (ref. 2020/9246) before it started, and was monitored by the Clinical Trial Unit of Rheumatology Service at Hospital del Mar. Due to the nature of the study (all the data are completely anonymous), the importance of expedited results, and their implication for treatment of patients during the SARS-COV-2 pandemic, we did not obtain informed consent from the participants. 


\section{Abbreviations}

ARBs: angiotensin II receptor blockers; ACE: angiotensin-converting-enzyme; $\mathrm{CI}$ : confidence interval; COVID-19: Coronavirus disease 19; CV: cardiovascular; NSAIDs: non-steroidal anti-inflammatory drugs; SERMs: selective estrogen receptor modulators; SSRIs: serotonin reuptake inhibitors.

\section{AUTHOR CONTRIBUTIONS}

J.B.R. was involved in conceptualizing the research idea, creating the research design, making the final interpretation of the statistical analyses, and writing the first draft and revision of the manuscript. N.S.D. participated in selection of statistical tests/analyses, performed the statistical analyses, computations and related computer work, and participated in writing the manuscript. L.T. was involved in conceptualizing the research idea, setting-up the research design, making the primary interpretation of the statistical analyses and participated in writing the manuscript. J.L.O. was involved in conceptualizing the research idea, setting-up the research design, making the primary interpretation of the statistical analyses and participated in writing the manuscript. M.C.R., L.P, and A.G. were responsible for patient cohort data collection. R.D.L.T. contributed to the statistical analyses and revised the manuscript. R.M. and J.M. were involved in conceptualizing the research idea, creating the research design, making the final interpretation of the statistical analysis, and writing the first draft and revision of the manuscript.

\section{ACKNOWLEDGMENTS}

We thank all patients who participated in the study, Gemma Vilagut $(\mathrm{PhD})$ for her guidance and support on the statistical analysis and Mònica Gratacós (PhD) for translating the clinical protocol into English, for English language support and proofreading of the manuscript. The Covidmar Study Group members are: Hospital del Mar, Barcelona: Selene Labrada, Miguel Mejía-Torres, Francisco Vilchez Oya, Antonio Meraz Ostiz, Helena Sirera Perelló (Rheumatology Service) and Irene Carrión-Barberà, Carolina Pérez-García, Fabiola Ojeda, Tarek Carlos Salman-Monte, Josep Blanch-Rubió, Anna Pros Simón, Emma Beltrán Catalán, Pedro Benito Ruiz (Rheumatology Service \& IMIM-Hospital del Mar Medical Research Institute) collected data and provided care for study patients; IMIM-Hospital del Mar Medical Research Institute: Luciano Polino, Laura Triginer, Anna Ribes (Cell Research on Inflammation and Cartillage Research Group, Inflammatory and Cardiovascular Processes Program) collected data; Maria-Victòria Puig (Integrative Pharmacology and Systems Neuroscience Research Group, Neurosciences
Research Program \& IMIM-Hospital del Mar Medical Research Institute); contributed to analysis design; Maria Teresa Martí Vila, Maria Luisa Perez Miras (CAP Vila Olímpica) collected data; Universitat Pompeu Fabra, Barcelona: Araceli Bergadà-Martínez, Cecilia Teresa Brambilla-Pisoni, Pablo Calvé- Pérez, Alba Calvet-Pavón, Laura Domingo-Rodríguez, Lorena Galera-López, Jolita Jančytė, Marta Linares-López, Elena Martín-García, Antonio Ortega-Álvaro, Pilar Ortiz-Teba, Sheila Piedra-Barrull, Dulce Real-Muñoz, Maria Sanchis-Ollé, Eric Senabre-Marchán, MiquelÀngel Serra, Anna Vázquez-Oliver (Laboratory of Neuropharmacology, Department of Experimental and Health Sciences \& IMIM-Hospital del Mar Medical Research Institute) collected data.

\section{CONFLICTS OF INTEREST}

All authors have completed the ICMJE uniform disclosure form at www.icmje.org/coi_disclosure.pdf, and declare: NSD has received funding from Centro de Información Cerveza y Salud (CICS); AG has received research grants or consulting fees from Astrazeneca and Bioiberica S.A.U.; RM has received research grants or consulting fees from Aelis, Almirall, Boehringer Ingelheim, BrainCo, Esteve, Ferrer, GlaxoSmithKline, Grünenthal, GW Pharmaceuticals, Janus, Lundbeck, Pharmaleads, Phytoplant, Rhodes, Sanofi, Spherium, Union de Pharmacologie Scientifique Appliquée, Upjohn, and Uriach; JM has received grants or consulting fees from Procare Health Iberia S.L, Esteve, Labhra, Bioibérica S.A.U, Grunenthal Pharma S.A, Pfizer, OPKO Heath Spain S.L.U and Roche Pharma S.A. MCR has received grants or consulting fees from Gedeon-Richter Ibérica, Gebro Pharma, Amgen S.A., Laboratorio Stada S.L., Lilly España S.A., Faes, Fidia, Theramex, Grunenthal; JBR has received grants or consulting fees from Amgen S.A., Laboratorio Stada S.L., Gedeon-Rhicter Ibérica, Lilly España, S.A., Pfizer, Gebro Pharma, and UCB Pharma S.A; LP has received grants from Lilly España, S.A y Celgene España S.L.; LT, JL-O and R.D.L.T. declare no competing interests.

\section{FUNDING}

This study was supported by Hospital del Mar. Authors funding includes "Ministerio de Ciencia, Innovación y Universidades" (\#AEI-SAF2017-84060-R FEDER to R.M., \#DPI2016-80283-C2-2-R), "Ministerio de Sanidad, Servicios Sociales e Igualdad" (\#RD16/0017/0020 \& \#PNSD-2017I068 to R.M., \#PI18/00059 to TCS-M) and "Generalitat de Catalunya" (\#2017-SGR-669 \& \#ICREA- Acadèmia 2015 to R.M., \#2017-SGR-138 to R.D.L.T.). N.S.D. is recipient of predoctoral fellowship \#2019-DI-47 from the DIUEAGAUR of the "Generalitat de Catalunya". 


\section{REFERENCES}

1. Huang $C$, Wang $Y$, Li $X$, Ren L, Zhao J, Hu Y, Zhang L, Fan G, Xu J, Gu X, Cheng Z, Yu T, Xia J, et al. Clinical features of patients infected with 2019 novel coronavirus in Wuhan, China. Lancet. 2020; 395:497-506. https://doi.org/10.1016/S0140-6736(20)30183-5 PMID:31986264

2. World Health Organization. World Health Organization. Coronavirus disease 2019 (COVID-19) [Internet]. Situation Report, 32. 2020. https://www.who.int/ docs/default-source/coronaviruse/situation-reports/ 20200505 covid-19-sitrep-106.pdf?sfursn $=47090 f 63$

3. Johns Hopkins Coronavirus Resource Center. COVID-19 dashboard by the Center for Systems Science and Engineering at Johns Hopkins University [Internet]. Johns Hopkins Coronavirus Resource Center. 2020. https://coronavirus.jhu.edu/map.html

4. Agencia de Salut Pública de Barcelona. Dades diaries de la infecció percoronavirus SARS Cov- 2 (COVID-19) a Barcelona [Internet]. 2020. https://www.aspb.cat/docs/COVID19aldiaBCN/

5. Cao Z, Li T, Liang L, Wang H, Wei F, Meng S, Cai M, Zhang Y, Xu H, Zhang J, Jin R. Clinical characteristics of coronavirus disease 2019 patients in Beijing, China. PLoS One. 2020; 15:e0234764.

https://doi.org/10.1371/journal.pone.0234764 PMID:32555674

6. Merad M, Martin JC. Pathological inflammation in patients with COVID-19: a key role for monocytes and macrophages. Nat Rev Immunol. 2020; 20:355-62.

https://doi.org/10.1038/s41577-020-0331-4 PMID:32376901

7. Khan F, Fabbri L, Stewart I, Robinson K, Smyth AR, Jenkins G. A systematic review of Anakinra, Tocilizumab, Sarilumab and Siltuximab for coronavirusrelated infections. medRxiv [Internet]. 2020. https://www.medrxiv.org/content/10.1101/2020.04.2 3.20076612v1?\%253fcollection= https://doi.org/10.1101/2020.04.23.20076612

8. Gianfrancesco M, Hyrich KL, Al-Adely S, Carmona L, Danila MI, Gossec L, Izadi Z, Jacobsohn L, Katz P, Lawson-Tovey S, Mateus EF, Rush S, Schmajuk G, et al, and COVID-19 Global Rheumatology Alliance. Characteristics associated with hospitalisation for COVID-19 in people with rheumatic disease: data from the COVID-19 global rheumatology alliance physicianreported registry. Ann Rheum Dis. 2020; 79:859-66. https://doi.org/10.1136/annrheumdis-2020-217871 PMID:32471903

9. Haberman R, Axelrad J, Chen A, Castillo R, Yan D, Izmirly P, Neimann A, Adhikari S, Hudesman D, Scher
JU. Covid-19 in immune-mediated inflammatory diseases - case series from New York. N Engl J Med. 2020; 383:85-88.

https://doi.org/10.1056/NEJMc2009567

PMID:32348641

10. Michelena X, Borrell H, López-Corbeto M, LópezLasanta M, Moreno E, Pascual-Pastor M, Erra A, Serrat M, Espartal E, Antón S, Añez GA, Caparrós-Ruiz R, Pluma A, et al. Incidence of COVID-19 in a cohort of adult and paediatric patients with rheumatic diseases treated with targeted biologic and synthetic diseasemodifying anti-rheumatic drugs. Semin Arthritis Rheum. 2020; 50:564-70.

https://doi.org/10.1016/i.semarthrit.2020.05.001 PMID:32425260

11. Consensus development conference: diagnosis, prophylaxis, and treatment of osteoporosis. Am J Med. 1993; 94:646-50.

https://doi.org/10.1016/0002-9343(93)90218-e PMID:8506892

12. Kanis JA, Cooper C, Rizzoli R, Reginster JY, and Scientific Advisory Board of the European Society for Clinical and Economic Aspects of Osteoporosis and Osteoarthritis (ESCEO) and the Committees of Scientific Advisors and National Societies of the International Osteoporosis Foundation (IOF). Executive summary of the European guidance for the diagnosis and management of osteoporosis in postmenopausal women. Calcif Tissue Int. 2019; 104:235-38.

https://doi.org/10.1007/s00223-018-00512-x PMID:30796490

13. American College of Rheumatology COVID-19 Guidance Task Force. COVID-19 Clinical Guidance for Patients with Rheumatic Diseases. Am Coll Rheumatol. 2020; $1-2$.

14. Landewé RB, Machado PM, Kroon F, Bijlsma HW, Burmester GR, Carmona L, Combe B, Galli M, Gossec L, lagnocco A, Isaacs JD, Mariette X, Mclnnes I, et al. EULAR provisional recommendations for the management of rheumatic and musculoskeletal diseases in the context of SARS-CoV-2. Ann Rheum Dis. 2020; 79:851-58.

https://doi.org/10.1136/annrheumdis-2020-217877 PMID:32503854

15. ASBMR webinar panel on treating patients with osteoporosis during the COVID-19 pandemic [Internet]. 2020. https://www.asbmr.org/educationdetail?cid=b92753f3-0a28-4f37-9a58-6ded595a7b40\#. XahFwy-ZM

16. Yu EW, Tsourdi E, Clarke BL, Bauer DC, Drake MT. Osteoporosis management in the era of COVID-19. J Bone Miner Res. 2020; 35:1009-13. https://doi.org/10.1002/ibmr.4049 PMID:32406536 
17. Xgeva (Denosumab). European Medicines Agency [Internet].

https://www.ema.europa.eu/en/medicines/human/EP AR/xgeva

18. Cheng ML, Fong L. Effects of RANKL-targeted therapy in immunity and cancer. Front Oncol. 2014; 3:329.

https://doi.org/10.3389/fonc.2013.00329

PMID:24432249

19. Kobayashi-Sakamoto M, Tamai R, Kiyoura Y. Beyond bone remodeling-emerging functions of osteoprotegerin in host defense and microbial infection. Integr Mol Med. 2015; 2:384-90. https://doi.org/10.15761/IMM.1000173

20. Wasserstein RL, Schirm AL, Lazar NA. Moving to a World Beyond "p < 0.05." Am Stat [Internet]. Taylor \& Francis; 2019; 73:1-19. https://doi.org/10.1080/00031305.2019.1583913

21. Amrhein V, Greenland S, McShane B. Scientists rise up against statistical significance. Nature. 2019; 567:305-307.

https://doi.org/10.1038/d41586-019-00857-9 PMID:30894741

22. Chiu YG, Ritchlin CT. Denosumab: targeting the RANKL pathway to treat rheumatoid arthritis. Expert Opin Biol Ther. 2017; 17:119-28.

https://doi.org/10.1080/14712598.2017.1263614 PMID:27871200

23. Baron R, Ferrari S, Russell RG. Denosumab and bisphosphonates: different mechanisms of action and effects. Bone. 2011; 48:677-92.

https://doi.org/10.1016/j.bone.2010.11.020

PMID:21145999

24. Cremers S, Drake MT, Ebetino FH, Bilezikian JP, Russell RG. Pharmacology of bisphosphonates. $\mathrm{Br} J$ Clin Pharmacol. 2019; 85:1052-62. https://doi.org/10.1111/bcp.13867 PMID: $\underline{30650219}$

25. Lyles KW, Colón-Emeric CS, Magaziner JS, Adachi JD, Pieper CF, Mautalen C, Hyldstrup L, Recknor C, Nordsletten L, Moore KA, Lavecchia C, Zhang J, Mesenbrink $\mathrm{P}$, et al, and HORIZON Recurrent Fracture Trial. Zoledronic acid and clinical fractures and mortality after hip fracture. N Engl J Med. 2007; 357:1799-809.

https://doi.org/10.1056/NEJMoa074941

PMID:17878149

26. Colón-Emeric CS, Mesenbrink P, Lyles KW, Pieper CF, Boonen S, Delmas P, Eriksen EF, Magaziner J. Potential mediators of the mortality reduction with zoledronic acid after hip fracture. J Bone Miner Res. 2010; 25:91-97. https://doi.org/10.1359/jbmr.090704 PMID:19580467
27. Bergman J, Nordström A, Hommel A, Kivipelto $M$, Nordström P. Bisphosphonates and mortality: confounding in observational studies? Osteoporos Int. 2019; 30:1973-82.

https://doi.org/10.1007/s00198-019-05097-1 PMID:31367949

28. Brufsky A, Marti JL, Nasrazadani A, Lotze MT. Boning up: amino-bisphophonates as immunostimulants and endosomal disruptors of dendritic cell in SARS-CoV-2 infection. J Transl Med. 2020; 18:261. https://doi.org/10.1186/s12967-020-02433-6 PMID: $\underline{32600410}$

29. Okamoto S, Jiang $Y$, Kawamura K, Shingyoji M, Tada Y, Sekine I, Takiguchi Y, Tatsumi K, Kobayashi H, Shimada $\mathrm{H}$, Hiroshima K, Tagawa M. Zoledronic acid induces apoptosis and $\mathrm{s}$-phase arrest in mesothelioma through inhibiting rab family proteins and topoisomerase II actions. Cell Death Dis. 2014; 5:e1517.

https://doi.org/10.1038/cddis.2014.475

PMID:25393473

30. Coxon FP, Helfrich MH, Van't Hof R, Sebti S, Ralston SH, Hamilton A, Rogers MJ. Protein geranylgeranylation is required for osteoclast formation, function, and survival: inhibition by bisphosphonates and GGTI-298. J Bone Miner Res. 2000; 15:1467-76.

https://doi.org/10.1359/jbmr.2000.15.8.1467 PMID:10934645

31. Poccia F, Agrati C, Castilletti C, Bordi L, Gioia C, Horejsh D, Ippolito G, Chan PK, Hui DS, Sung JJ, Capobianchi MR, Malkovsky M. Anti-severe acute respiratory syndrome coronavirus immune responses: the role played by $\mathrm{V}$ gamma $9 \mathrm{~V}$ delta $2 \mathrm{~T}$ cells. J Infect Dis. 2006; 193:1244-49. https://doi.org/10.1086/502975 PMID:16586361

32. El-Kurdi B, Khatua B, Rood C, Snozek C, Cartin-Ceba R, Singh VP, and Lipotoxicity in COVID-19 Study Group. Mortality from coronavirus disease 2019 increases with unsaturated fat and may be reduced by early calcium and albumin supplementation. Gastroenterology. 2020; 159:1015-18.e4.

https://doi.org/10.1053/i.gastro.2020.05.057 PMID:32470338

33. Cappellini F, Brivio R, Casati M, Cavallero A, Contro E, Brambilla P. Low levels of total and ionized calcium in blood of COVID-19 patients. Clin Chem Lab Med. 2020; 58:e171-73.

https://doi.org/10.1515/cclm-2020-0611 PMID:32459190

34. Vaeth M, Eckstein M, Shaw PJ, Kozhaya L, Yang J, Berberich-Siebelt F, Clancy R, Unutmaz D, Feske S. Store-operated $\mathrm{Ca}(2+)$ entry in follicular T cells controls humoral immune responses and autoimmunity. Immunity. 2016; 44:1350-64. 
https://doi.org/10.1016/i.immuni.2016.04.013

PMID:27261277

35. Martino $M$, Rocchi $G$, Escelsior $A$, Fornaro $M$. Immunomodulation mechanism of antidepressants: interactions between serotonin/norepinephrine balance and Th1/Th2 balance. Curr Neuropharmacol. 2012; 10:97-123.

https://doi.org/10.2174/157015912800604542

PMID:23204981

36. Brunton LL. Goodman and Gilman's: The Pharmacological Basis of Therapeutics. 13th ed. 2018.

37. Buschmann HH, Hernandez JMV. Use of compounds binding to the sigma receptor for the treatment of metabolic syndrome. Spain; EP 1787679 A1, 2007.

38. Gordon DE, Jang GM, Bouhaddou M, Xu J, Obernier K, White KM, O'Meara MJ, Rezelj VV, Guo JZ, Swaney DL, Tummino TA, Hüttenhain R, Kaake RM, et al. A SARSCoV-2 protein interaction map reveals targets for drug repurposing. Nature. 2020; 583:459-68.

https://doi.org/10.1038/s41586-020-2286-9

PMID:32353859

39. Ortuño MJ, Robinson ST, Subramanyam $P$, Paone $R$, Huang YY, Guo XE, Colecraft HM, Mann JJ, Ducy P. Serotonin-reuptake inhibitors act centrally to cause bone loss in mice by counteracting a local antiresorptive effect. Nat Med. 2016; 22:1170-79. https://doi.org/10.1038/nm.4166 PMID:27595322

40. Taylor CP, Angelotti T, Fauman E. Pharmacology and mechanism of action of pregabalin: the calcium channel alpha2-delta (alpha2-delta) subunit as a target for antiepileptic drug discovery. Epilepsy Res. 2007; 73:137-50.

https://doi.org/10.1016/j.eplepsyres.2006.09.008

PMID:17126531
41. Abu-Rish EY, Mansour AT, Mansour HT, Dahabiyeh LA, Aleidi SM, Bustanji Y. Pregabalin inhibits in vivo and in vitro cytokine secretion and attenuates spleen inflammation in Lipopolysaccharide/Concanavalin A induced murine models of inflammation. Sci Rep. 2020; 10:4007.

https://doi.org/10.1038/s41598-020-61006-1

PMID:32132609

42. Lee $K$, Vivithanaporn $P$, Siemieniuk RA, Krentz $H B$, Maingat F, Gill MJ, Power C. Clinical outcomes and immune benefits of anti-epileptic drug therapy in HIV/AIDS. BMC Neurol. 2010; 10:44.

https://doi.org/10.1186/1471-2377-10-44

PMID:20565780

43. Awwad ZM, El-Ganainy SO, ElMallah Al, Khedr SM, Khattab MM, El-Khatib AS. Assessment of pregabalininduced cardiotoxicity in rats: mechanistic role of angiotensin 1-7. Cardiovasc Toxicol. 2020; 20:301-11.

https://doi.org/10.1007/s12012-019-09553-6 PMID:31720995

44. Santos RA, Sampaio WO, Alzamora AC, Motta-Santos D, Alenina N, Bader M, Campagnole-Santos MJ. The ACE2/Angiotensin-(1-7)/MAS axis of the reninangiotensin system: focus on angiotensin-(1-7). Physiol Rev. 2018; 98:505-53.

https://doi.org/10.1152/physrev.00023.2016 PMID:29351514

45. Zhang $X, Y u J$, Pan LY, Jiang HY. ACEI/ARB use and risk of infection or severity or mortality of COVID-19: A systematic review and meta-analysis. Pharmacol Res. 2020; 158.

https://doi.org/10.1016/j.phrs.2020.104927

PMID:32422341 
Supplementary Table 3. Relative risk (RR) and 95\% confidence interval ( $95 \% \mathrm{Cl}$ ) of hsCOVID-19 after propensity score matching.

\begin{tabular}{|c|c|c|c|c|c|c|c|c|c|}
\hline \multirow{2}{*}{ Sample size } & \multicolumn{3}{|c|}{ Denosumab } & \multicolumn{3}{|c|}{ Intravenous Zoledronate } & \multicolumn{3}{|c|}{ Calcium } \\
\hline & & control & treated & & control & treated & & control & treated \\
\hline & N All & 1838 & 264 & N All & 1923 & 179 & N All & 1612 & 490 \\
\hline & $\mathrm{N}$ matched & 264 & 264 & $\mathrm{~N}$ matched & 179 & 179 & $\mathrm{~N}$ matched & 490 & 490 \\
\hline & $\mathrm{N}$ unmatched & 1574 & 0 & $\mathrm{~N}$ unmatched & 1744 & 0 & $\mathrm{~N}$ unmatched & 1122 & 0 \\
\hline \multirow{2}{*}{$\begin{array}{l}\text { COVID-19 (matched } \\
\text { data) }\end{array}$} & Yes & 11 & 8 & Yes & 11 & 6 & Yes & 22 & 16 \\
\hline & No & 253 & 256 & No & 168 & 173 & No & 468 & 474 \\
\hline Crude RR $(95 \%$ CI $)$ & \multicolumn{3}{|c|}{$0.73(0.30-1.78)$} & \multicolumn{3}{|c|}{$0.55(0.20-1.44)$} & \multicolumn{3}{|c|}{$0.72(0.39-1.37)$} \\
\hline Adjusted RR $(95 \% \text { CI })^{*}$ & \multicolumn{3}{|c|}{$0.87(0.30-2.52)$} & \multicolumn{3}{|c|}{$0.43(0.14-1.35)$} & \multicolumn{3}{|c|}{$0.67(0.36-1.27)$} \\
\hline Summary of balance & Means & Means & Mean Diff & Means & Means & Mean Diff & Means & Means & Mean \\
\hline for matched data & Treated & Control & & Treated & Control & & Treated & Control & Diff \\
\hline Osteoporosis & 0.97 & 0.97 & 0.00 & 0.92 & 0.92 & 0.00 & 0.77 & 0.77 & 0.00 \\
\hline Women & 0.86 & 0.86 & -0.01 & 0.77 & 0.80 & -0.03 & 0.85 & 0.88 & -0.02 \\
\hline Age & 74.05 & 73.29 & 0.76 & 71.61 & 71.69 & -0.08 & 71.34 & 71.57 & -0.22 \\
\hline CV disease & 0.17 & 0.13 & 0.04 & 0.18 & 0.17 & 0.02 & 0.17 & 0.16 & 0.01 \\
\hline Diabetes & 0.12 & 0.10 & 0.02 & 0.18 & 0.17 & 0.01 & 0.10 & 0.12 & -0.02 \\
\hline Pulmonary disease & 0.20 & 0.21 & -0.01 & 0.19 & 0.17 & 0.02 & 0.16 & 0.14 & 0.01 \\
\hline Renal disease & 0.11 & 0.09 & 0.01 & 0.08 & 0.04 & 0.03 & 0.07 & 0.06 & 0.01 \\
\hline Cancer & 0.09 & 0.08 & 0.02 & 0.15 & 0.15 & 0.01 & 0.08 & 0.08 & 0.00 \\
\hline
\end{tabular}

$\mathrm{CV}=$ cardiovascular.

*RR for denosumab is adjusted by the exposure to calcium, bisphosphonates, vitamin $D$, thiazide, analgesics, dual antidepressants, tricyclic antidepressants, SSRI antidepressants (selective serotonin reuptake inhibitors), glucocorticoids, anti-hypertensive drugs and NSAIDs. RR for intravenous zoledronate is adjusted by the exposure to denosumab, oral bisphosphonates, calcium, vitamin D, thiazide, analgesics, dual antidepressants, tricyclic antidepressants, SSRI antidepressants, glucocorticoids, anti-hypertensive drugs and NSAIDs. RR for calcium is adjusted by the exposure to denosumab, bisphosphonates, vitamin D, thiazide, analgesics, dual antidepressants, tricyclic antidepressants, SSRI antidepressants, glucocorticoids, anti-hypertensive drugs and NSAIDs. 